\title{
Maintenance of antioxidant defenses of brain cells: plasma membrane glutamate transporters and beyond
}

\author{
Laurence Had-Aissouni
}

Received: 20 December 2010/Accepted: 17 February 2011/Published online: 11 March 2011

(C) Springer-Verlag 2011

Oxidative stress is invariably involved in neurodegenerative insults as a contributing mechanism in various forms of cell death (Bains and Shaw 1997). If strategies have to be developed for protection against oxidative injury, a better understanding of how antioxidant defenses are maintained in the brain is required. In this issue, leading scientists in the field present the state of the art concerning how precursors of the main brain antioxidant glutathione are delivered to brain cells, and explore regulatory mechanisms to identify new neuroprotective strategies against neurodegenerative insults. The essence of these articles comes from lectures presented at the 11th International Congress on Amino Acids, Peptides and Proteins held in Vienna, August 3-7, 2009. The meeting was organized by Pr. G. Lubec and the session on glutamate, its transporters and antioxidant defenses by Dr. L. Had-Aissouni.

Glutathione (GSH) is the main brain antioxidant molecule. It is a tripeptide composed of glutamate, cysteine and glycine. Substrates for GSH synthesis may be provided by direct transport of constituent amino acids from the extracellular milieu. In this context, cysteine is thought to be the limiting precursor for GSH synthesis. Indeed in the extracellular milieu it is oxidized into cystine and intracellularly (in the reduced form, cysteine) it is present at a lower concentration, compared to the other constituent of GSH in most cells. However, none of these amino acids is indispensable, so they can also be synthesized in cells from other amino acids, providing that the necessary metabolic pathways are present (McBean 2011). Moreover, released

L. Had-Aissouni $(\bowtie)$

Developmental Biology Institute of Marseille-Luminy,

CNRS/Aix-Marseille University, Luminy Campus-Case 907,

13288 Marseille cedex 9, France

e-mail: laurence.had-aissouni@univmed.fr
GSH or conjugates are substrates for the ectoenzyme gamma-glutamyltransferase (EC 2.3.2.2) forming dipeptides such as cysteinylglycine or gamma-glutamylcysteine that, after extracellular hydrolysis, may provide substrates for GSH synthesis. Dipeptides may also be transported intracellularly in some cells and in the case of gammaglutamylcysteine used to bypass the first step of GSH synthesis (Dringen 2000). Due to the different pathways that cells may use to obtain substrates for GSH synthesis, it is very unlikely that the activity of a single amino acid transport system is required to sustain GSH synthesis. However, data obtained in culture as well as in vivo suggest that plasma membrane glutamate transporters may be a determinant for maintenance of antioxidant defenses in the brain.

Before cloning of the cDNA coding for amino acid transporter proteins or subunits, transport systems were identified and characterized according to functional criteria such as transport mechanism (exchange, uniport, cotransport, counter transport, primary or secondary active transport) substrate specificity (that is generally rather large) and ionic dependence (mainly sodium dependent or independent). These characteristics were first used to name the corresponding transport systems generally using the first letter of the preferred substrate or $\mathrm{x}$ for anionic amino acids or $\mathrm{y}$ for cationic amino acids, sodium-dependent transport systems being designated in upper case while sodium-independent transport system are generally designated in lower case. Moreover, the charge of the transported substrates may also be indicated in superscript $(0,+$, or -$)$ while the first letter of the preferred substrate may be indicated in subscript when not directly used to name the transport system. According to this nomenclature, glutamate was found to be transported inside cells in sodium-independent as well as sodium-dependent 
pathways by two transport systems for anionic amino acid, named system $\mathrm{x}_{\mathrm{C}}^{-}$and system $\mathrm{X}_{\mathrm{AG}}^{-}$, respectively (Christensen et al. 1994).

System $\mathrm{x}_{\mathrm{C}}$ is able to transport both cystine and glutamate in exchange of each other in a sodium-independent but chloride-dependent way and transports cystine in exchange of intracellular glutamate with an 1:1 ratio in physiological conditions. Glutamate uptake by this system is inhibited by cystine, quisqualate, ibotenate, $\alpha$-aminoadipate and (S)-4-carboxyphenylglycine (4-CPG), but not by aspartate; 4-CPG, originally identified as an agonist/ antagonist of metabotropic glutamate receptors, being a blocker of system $x_{\bar{C}}^{-}$(Patel et al. 2004). The transporter responsible for this uptake has now been identified by molecular cloning (Conrad and Sato 2011). It belongs to the heteromeric amino acid transporter (HAT) family and consists of two different subunits: the 4F2 heavy chain/ CD98 surface antigen and a protein of 502 amino acid residues named $\mathrm{xCT}$, for system $\mathrm{x}_{\overline{\mathrm{C}}}^{-}$transporter protein, as this subunit is responsible for substrate specificity. System $\mathrm{x}_{\mathrm{C}}^{-}$is the first transport system whose activity in astrocytes and immature neurons in culture was found to be essential for maintaining GSH levels. Transported cystine is rapidly reduced to cysteine in the cytoplasm and is thought to be the better donor of cysteine moiety for GSH synthesis. Indeed, when cystine uptake by system $\mathrm{x}_{\mathrm{C}}^{-}$is inhibited by high extracellular glutamate concentrations, the subsequent severe deficiency in GSH may lead to cell degeneration through oxidative glutamate toxicity (Lewerenz et al. 2011).

Excitatory amino acid transporters (EAATs) form the system $\mathrm{X}_{\mathrm{AG}}^{-}$which transports anionic amino acid while three $\mathrm{Na}^{+}$and one $\mathrm{H}^{+}$are co-transported and one $\mathrm{K}^{+}$is counter-transported and are, therefore, secondary active depending on the ionic gradients maintained by the sodium/potassium pump (Danbolt 2001). This system demonstrates a high affinity for L-glutamate and L- and D-aspartate but also for cyst(e)ine (Hayes et al. 2005; Zerangue and Kavanaugh 1996). It is the second transport system found to provide precursors for GSH synthesis. In glial cells, EAAT transported glutamate may either be directly used as a substrate for GSH or help to transport cystine through its release by system $\mathrm{x}_{\mathrm{C}}^{-}$(Gras et al. 2011; Had-Aissouni 2011; Persson and Rönnbäck 2011). In neuronal cells EAATs are also important but it seems to be because neuronal EAATs are the main entry route for cysteine into these cells (Aoyama et al. 2011). Therefore, if it can be clearly stated that EAATs are important for maintaining GSH levels, the substrate they provide for fulfilling this function may be different according to cell type. The relative importance of EAATs and $\mathrm{x}_{\mathrm{C}}^{-}$in maintaining GSH levels has also been tested in vivo in knockout mice. There is surprisingly no GSH deficiency in $\mathrm{xCT}$ deficient mice and a novel function has been recently attributed to system $\mathrm{x}_{\mathrm{C}}^{-}$in basal conditions. It may participate in a cystine-cysteine cycle important to maintain the external redox balance and limit lipid peroxidation (Conrad and Sato 2011). On the contrary there is a delayed GSH depletion in brain from mice deficient in EAAT3/EAAC1 (excitatory amino acid carrier 1), the main neuronal EAAT, (Aoyama et al. 2011) and there is a GSH deficiency in retinal glial cells of mice deficient in one of the glial EAATs, EAAT1/GLAST (glutamate aspartate transporter), (Harada et al. 2007).

Following the cloning of the whole human genome, the Human Genome Organization Nomenclature Committee has chosen to regroup all the genes coding for membrane transporters in the solute carrier (SLC) gene series. Fortysix families and 360 transporter genes are included in these series (Hediger et al. 2004). EAAT genes belong to the Slcla family along with two genes coding for the neutral amino acid transporters ASCT1 and 2 (alanine, serine, cysteine-preferring transporters). Therefore, the EAAT3/ EAAC1 gene is called Slclal; the EAAT2/GLT-1 (glutamate transporter-1) gene, Slcla2; the EAAT1/GLAST gene, Slcla3; the EAAT4 gene, Slcla6 and the EAAT5 gene, Slcla7 (Kanai and Hediger 2004). The heavy chain of $\mathrm{x}_{\mathrm{C}}^{-}(4 \mathrm{~F} 2 \mathrm{hc})$ is encoded by the gene Slc3a2 (Palacin and Kanai 2004) and the light chain xCT by the gene Slc7all (Verrey et al. 2004). Due to the complexity of the nomenclature, EAAC1, EAAT4 and EAAT5 will be used to describe the neuronal EAATs, GLT-1 and GLAST will be used to describe the glial EAATs and system $\mathrm{x}_{\mathrm{C}}^{\overline{\mathrm{C}}}$ to describe the HAT that transports extracellular cystine in exchange for intracellular glutamate.

It is important to note that both types of transporters (EAATs and system $\mathrm{x}_{\mathrm{C}}^{-}$) contribute to glutamate homeostasis in the brain. In this organ, glutamate is not only a metabolite but also a signaling molecule used by most excitatory neurons. Moreover, at high extracellular concentrations glutamate is a potent neurotoxin that may trigger neuronal death through over-stimulation of glutamatergic receptors, a process named excitotoxicity. Therefore, extracellular glutamate concentrations must be maintained below the micromolar range to maintain the phasic aspect of neurotransmission and prevent excitotoxic neuronal death as well as inhibition of system $\mathrm{x}_{\mathrm{C}}$ and oxidative glutamate toxicity (Danbolt 2001). Stimulation of system $\mathrm{x}_{\mathrm{C}}^{-}$activity, following oxidative injury or amino acid depletion (Lewerenz et al. 2011), if not compensated by over-activity of EAATs, as well as the sole EAAT impairment, as observed in neurodegenerative insults, may induce extracellular glutamate concentrations to build up and reach toxic levels. Such dysfunctions may also deplete intracellular glutamate, impair GSH synthesis and contribute to another form of oxidative toxicity due to low 
intracellular glutamate (Had-Aissouni 2011). Therefore, a better understanding of how the activities of both types of transporters are regulated and even coordinated is required to be able to design intervention strategies against these different and intricate toxic processes. The review articles in this issue are focused on the mechanisms used by the different brain cell types to obtain glutathione precursors, their importance for cells in health and disease, and the exploration of new and promising perspectives for protection in neurodegenerative insults. Many thanks are due to all authors for their contributions and to Pr. G. Lubec, his collaborators and supporting institutions for organizing the 11th International Congress on Amino Acids, Peptide and Proteins.

\section{References}

Aoyama K, Watabe M, Nakaki T (2011) Modulation of neuronal glutathione synthesis by EAAC1 and its interacting protein GTRAP3-18. Amino acids (in this issue). doi:10.1007/s00726011-0861-y

Bains JS, Shaw CA (1997) Neurodegenerative disorders in humans: the role of glutathione in oxidative stress-mediated neuronal death. Brain Res Brain Res Rev 25:335-358

Christensen HN, Albritton LM, Kakuda DK et al (1994) Geneproduct designations for amino acid transporters. J Exp Biol 196:51-57

Conrad M, Sato H (2011) The oxidative stress-inducible cystine/ glutamate antiporter, system $\mathrm{x}_{\mathrm{c}}^{-}$: cystine supplier and beyond. Amino acids (in this issue). doi:10.1007/s00726-011-0867-5

Danbolt NC (2001) Glutamate uptake. Prog Neurobiol 65:1-105

Dringen R (2000) Metabolism and functions of glutathione in brain. Prog Neurobiol 62:649-671

Gras G, Samah B, Hubert A et al (2011) EEAT expression by macrophages and microglia: still more question than answers. Amino acids (in this issue). doi:10.1007/s00726-011-0866-6
Had-Aissouni L (2011) Toward a new role for plasma membrane sodium-dependent glutamate transporters of astrocytes: maintenance of antioxidant defenses beyond extracellular glutamate clearance. Amino acids (in this issue). doi:10.1007/s00726011-0863-9

Harada T, Harada C, Nakamura K et al (2007) The potential role of glutamate transporters in the pathogenesis of normal tension glaucoma. J Clin Invest 117:1763-1770

Hayes D, Wiessner M, Rauen $\mathrm{T}$ et al (2005) Transport of $\mathrm{L}-\left[{ }^{14} \mathrm{C}\right]$ cystine and $\mathrm{L}-\left[{ }^{14} \mathrm{C}\right]$ cysteine by subtypes of high affinity glutamate transporters over-expressed in HEK cells. Neurochem Int 46:585-594

Hediger MA, Romero MF, Peng JB et al (2004) The ABCs of solute carriers: physiological, pathological and therapeutic implications of human membrane transport proteins: Introduction. Pflugers Arch 447:465-468

Kanai Y, Hediger MA (2004) The glutamate/neutral amino acid transporter family SLC1: molecular, physiological and pharmacological aspects. Pflugers Arch 447:469-479

Lewerenz J, Maher P, Methner A (2011) Regulation of xCT expression and system $\mathrm{x}_{\mathrm{c}}^{-}$function in neuronal cells. Amino acids (in this issue). doi:10.1007/s00726-011-0862-x

McBean G (2011) The transsulfuration pathway: a source of cysteine for glutathione in astrocytes. Amino acids (in this issue). doi:10.1007/s00726-011-0864-8

Palacin M, Kanai Y (2004) The ancillary proteins of HATs: SLC3 family of amino acid transporters. Pflugers Arch 447:490-494

Patel SA, Warren BA, Rhoderick JF et al (2004) Differentiation of substrate and non-substrate inhibitors of transport system xc(-): an obligate exchanger of L-glutamate and L-cystine. Neuropharmacology 46:273-284

Persson M, Rönnbäck L (2011) Microglial self defence mediated through GLT-1 and glutathione. Amino acids (in this issue). doi:10.1007/s00726-011-0865-7

Verrey F, Closs EI, Wagner CA et al (2004) CATs and HATs: the SLC7 family of amino acid transporters. Pflugers Arch 447:532-542

Zerangue N, Kavanaugh MP (1996) Interaction of L-cysteine with a human excitatory amino acid transporter. J Physiol 493:419-423 